\title{
APPROXIMATION BY DOUBLE WALSH POLYNOMIALS
}

\author{
FERENC MÓRICZ \\ University of Szeged \\ Bolyai Institute \\ Aradi vértanák tere 1 \\ 6720 Szeged, Hungary \\ (Received June 20, 1991)
}

ABSTRACT. We study the rate of approximation by rectangular partial sums, Cesàro means, and de la Vallée Poussin means of double Walsh-Fourier series of a function in a homogeneous Banach space $X$. In particular, $X$ may be $L^{p}\left(I^{2}\right)$, where $1 \leqq F<\infty$ and $I^{2}=[0,1) \times[0,1)$, or $C_{W}\left(I^{2}\right)$, the latter being the collection of uniformly $W$-continuous functions on $I^{2}$. We extend the results by Watari, Fine, Yano, Jastrebova, Bljumin, Esfahanizadeh and Siddiqi from univariate to multivariate cases. As by-products, we deduce sufficient conditions for convergence in $L^{p}\left(I^{2}\right)$-norm and uniform convergence on $I^{2}$ as well as characterizations of Lipschitz classes of functions. At the end, we raise three problems.

KEY WORDS AND PHRASES. Walsh-Paley system, homogeneous Banach space, best approximation, $W$-continuity, modulus of continuity, Lipschitz class, rectangular partial sum, Cesàro mean, de la Vallée poussin mean, Dirichlet kernel, Fejér kernel, convergence in $L^{p}$-norm, uniform convergence, saturation problem.

1980 AMS SUBJECT CLASSIFICATION CODE.

Primary 41A50, Secondary 42C10, 40G05.

1. INTRODUCTION.

We consider the Walsh orthonormal system $\left\{w_{j}(x): j \geqq 0\right\}$ defined on the unit interval $I:=[0,1)$ in the Paley enumeration (see [7]). To be more specific, let

$$
\begin{aligned}
& r_{0}(x):=\left\{\begin{array}{lll}
1 & \text { if } & x \in\left[0,2^{-1}\right), \\
-1 & \text { if } & x \in\left[2^{-1}, 1\right),
\end{array}\right. \\
& r_{0}(x+1):=r_{0}(x), \\
& r_{j}(x):=r_{0}\left(2^{j} x\right), \quad j \geq 1 \text { and } x \in I,
\end{aligned}
$$

be the well-known Rademacher functions. For $k=0$ set $w_{0}(x):=1$, and if

$$
k:=\sum_{j=0}^{\infty} k_{j} 2^{j}, k_{j}=0 \text { or } 1,
$$


is the dyadic representation of an integer $k \geqslant 1$, then set

$$
w_{k}(x):=\prod_{j=0}^{\infty}\left[r_{j}(x)\right]^{k} \text {. }
$$

We will study approximation by means of double Walsh polynomials in the norm of a homogeneous Banach space $X$ of functions defined on the unit square $I^{2}:=[0,1) \times[0,1)$.

2. DOUBLE WALSH POLYNOMIALS AND MODULUS OF CONTINUITY.

We remind the reader that a double walsh polynomial of order less than $m$ in $x$ and of order less than $n$ in $y$ is a two variable function of the form

$$
P(x, y):=\sum_{j=0}^{m-1} \sum_{k=0}^{n-1} a_{j k} w_{j}(x) w_{k}(y)
$$

where $m, n$ are positive integers and $\left\{a_{j k}\right\}$ is a double sequence of real (or complex) numbers. Denote by $P_{m n}$ the collection of such walsh polynomials and let

$$
P:=\bigcup_{m=1}^{\infty} \bigcup_{n=1}^{\infty} P_{m n} .
$$

The members of $P$ are called double Walsh polynomials.

Denote by $\Sigma_{m n}$ the finite $\sigma$-algebra generated by the collection of dyadic intervals of the form $I_{m n}(j, k):=\left[j 2^{-m},(j+1) 2^{-m}\right) \times\left[k 2^{-n},(k+1) 2^{-n}\right)$ where $0 \leqq j<2^{m}, 0 \leqq k<2^{n}$, and $m, n \geq 0$. It is plain that the collection of $\Sigma_{m n}$-measurable functions defined on $I^{2}$ coincides with $\mathrm{P}_{2^{m}}, 2^{n}$. The so-called dyadic topology of $I^{2}$ is generated by the union of the $\Sigma_{m n}$ for $m, n=0,1, \ldots$ The definition of a homogeneous Banach space on the circle group $T=[-\pi, \pi)$ is well-known (see Katznelson [6]). It is formulated on the dyadic group $I=[0,1)$, while using Walsh polynomials (see Butzer and Nessel [2] and also [8,pp. 154-155]). Following them, we say that a Banach space $X$ of functions defined on $I^{2}$ with the norm $\mid \|_{X}$ is homogeneous if $P \subseteq X \subseteq L^{1}\left(I^{2}\right)$ and if the following three properties hold:

(i) The norm of $X$ dominates the $L^{1}\left(I^{2}\right)$-norm: for any $f \in X$

$$
\|f\|_{1} \leqq\|f\|_{X} \text {; }
$$

(ii) The norm of $X$ is translation invariant: for any $(u, v) \in I^{2}$ and $f \in X$

$$
\tau_{u v} f \in X \text { and }\left\|\tau_{u v} f\right\|_{X}=\|f\|_{X}
$$

where $\tau_{u v}$ means the dyadic translation by $u$ in the first variable and by $v$ in the second one:

$$
\tau_{u v} f(x, y):=f(x+u, y+v), \quad(x, y) \in I^{2} \text {. }
$$

Here and in the sequel, $\dot{+}$ denotes dyadic addition.

(iii) $P$ is dense in $X$ with respect to the norm $\mid \|^{\prime}$, i.e., for any $f \in X$ and $\varepsilon>0$ there exists a double walsh polynomial $P \in P$ such that 


$$
\|P-f\|_{X} \leqq \varepsilon .
$$

We recall that the norm in $L^{p}\left(I^{2}\right), 1 \leq p<\infty$, is defined by

$$
\|f\|_{p}:=\left\{\begin{array}{ll}
1 & 1 \\
\int & \int_{0}|f(x, y)|^{p} d x d y
\end{array}\right\}^{1 / p} \text {. }
$$

while $C_{W}\left(I^{2}\right)$ is the collection of functions $f(x, y)$ that are uniformly continuous from the dyadic topology of $I^{2}$ to the usual topology of $R$, and endowed with the "sup" norm:

$$
\|f\|_{\infty}:=\sup \left\{|f(x, y)|:(x, y) \in I^{2}\right\} .
$$

Such a function $f$ is called uniformly $W$-continuous.

Similarly to the univariate case (cf. [8, pp. 9-11]) if the periodic extension of a function $f(x, y)$ from $I^{2}$ to $R^{2}$ with period 1 in both $x$ and $y$ is classically continuous, then $f$ is also uniformly $W-$ continuous on $I^{2}$.

It follows (cf. [8, p. 142] in the univariate case) that $L^{p}\left(I^{2}\right)$ is the closure of the collection $P$ of double Walsh polynomials when using the norm $\|\cdot\|_{p}, 1 \leqq p<\infty$. Likewise (cf. [8, pp. 156-158]), $C_{W}\left(I^{2}\right)$ is the uniform closure of $P$, i.e., when using the norm $|\cdot|_{\infty}$. The extension of [8, Lemma $1, p .155]$ from $I$ to $I^{2}$ is of basic importance in this paper.

LEMMA 1. For any $f, h \in X$ and $g \in L^{1}\left(I^{2}\right)$

$$
\begin{aligned}
& \| f^{*} g-\left.h \int_{0}^{1} \int_{0}^{1} g(u, v) d u d v\right|_{X} \\
& \leqq \int_{0}^{1} \int_{0}^{1}\left|\tau_{u v} f-h\right|_{X}|g(u, v)| d u d v
\end{aligned}
$$

where

$$
(f * g)(x, y):=\int_{0}^{1} \int_{0}^{1} f(x+u, y \dot{+} v) g(u, v) d u d v, \quad(x, y) \in I^{2},
$$

is the dyadic convolution of the functions $f$ and $g$.

The proof of Lemma 1 is almost identical to that of the univariate lemma in [8, pp. 155-156]. We omit it.

Finally, we remind the reader that the (total) modulus of continuity of a function $f \in X$ is defined by

$$
{ }^{\omega_{X}}\left(f ; \delta_{1}, \delta_{2}\right):=\sup \left\{\mathbf{I}_{u v} f-f \mathbf{I}_{X}: 0 \leq u<\delta_{1}, 0 \leq v<\delta_{2}\right\}
$$

where $\delta_{1}, \delta_{2}>0$. By the Banach-Steinhaus theorem, for any $f \in X$

$$
\lim _{u, v \rightarrow 0} \mid \tau_{u v} f-f \|_{X}=0 \text {, }
$$

and consequently,

$$
\lim _{\delta_{1}, \delta_{2} \rightarrow 0}{ }^{\omega} X\left(f ; \delta_{1}, \delta_{2}\right)=0
$$

For $\alpha, \beta>0$, the Lipschitz class is defined by

$$
\operatorname{Lip}(\alpha, \beta ; X):=\left\{f \in X: \omega_{X}\left(f ; \delta_{1}, \delta_{2}\right)=O\left(\delta_{1}^{\alpha}+\delta_{2}^{\beta}\right) \text { as } \delta_{1}, \delta_{2} \rightarrow 0\right\} \text {. }
$$

Unlike the classical case, $\operatorname{Lip}(\alpha, \beta ; X)$ is not trivial when $\alpha>1$ and/or B > 1 (cf. [8, p. 188]). 
3. APPROXIMATION BY RECTANGULAR PARTIAL SUMS.

As is well-known, the measurement of the rate of approximation to a function $f \in X$ by polynomials in $P_{m n}$ is defined by

$$
E_{m n}(f ; X):=\inf \left\{\|f-P\|_{X}: P \in P_{m n}\right\} \text {. }
$$

Since $P_{m n}$ is a finite dimensional subspace of $X$, for every $f \in X$ the infimum above is attained by some $P_{m n} \in P_{m n}$. Such a polynomial $P_{m n}$ is called a best approximation of $f$ in $P_{m n}$.

Given a function $f \in L^{1}\left(I^{2}\right)$, we form its double walsh-Fourier series as follows

$$
\sum_{j=0}^{\infty} \sum_{k=0}^{\infty} a_{j k} w_{j}(x) w_{k}(y)
$$

where the

$$
a_{j k}:=\int_{0}^{1} \int_{0}^{1} f(u, v) w_{j}(u) w_{k}(v) d u d v, \quad j, k \geqq 0,
$$

are called double walsh-Fourier coefficients of $f$. The rectangular partial sums of series (3.1) are defined by

$$
S_{m n}(f ; x, y):=\sum_{j=0}^{m-1} \sum_{k=0}^{n-1} a_{j k} w_{j}(x) w_{k}(y), \quad m, n \geq 1 .
$$

Now, the modulus of continuity gives sharp estimates to the rate of approximation by double Walsh polynomials $P \in P\left(2^{m}, 2^{n}\right.$ and by the rectangular partial sums $S_{2^{m}, 2^{n}}(f)$.

THEOREM 1. For any $f \in X$ and $m, n \geqq 0$,

$$
\begin{aligned}
& 2^{-1} \omega_{X}\left(f ; 2^{-m}, 2^{-n}\right) \leq E_{2^{m}, 2^{n}}(f ; X) \\
& \leqq S_{2^{m}, 2^{n}}(f)-f \|_{X} \leq \omega_{X}\left(f ; 2^{-m}, 2^{-n}\right) .
\end{aligned}
$$

We note that the right inequality is the walsh analogue of the classical Jackson inequality. The left-most inequality has no trigonometric analogue.

PROOF. As is well-known,

$$
S_{m n}(f ; x, y)=\int_{0}^{1} \int_{0}^{1} f(x \dot{+} u, y \dot{+} v) D_{m}(u) D_{n}(v) d u d v
$$

where

$$
D_{m}(u):=\sum_{j=0}^{m-1} w_{j}(u), \quad m \geqq 1,
$$

is the Walsh-Dirichlet kernel. We recall that the Paley lemma (see, e.g., [8, p 7]) says that

$$
D_{2^{m}}(u)=\left\{\begin{array}{lll}
2^{m} & \text { if } & u \in\left[0,2^{-m}\right), \\
0 & \text { if } & u \in\left[2^{-m}, 1\right) .
\end{array}\right.
$$

Now, by (2.1), 


$$
\begin{aligned}
\left\|S 2^{m}, 2^{n}(f)-f\right\|_{X} & \leq \int_{0}^{1} \int_{0}^{1}\left\|_{u v} f-f\right\|_{X}{ }_{2}(u) D_{2}(v) d u d v \\
& =2^{m+n} \int_{0} \int_{0}^{-m} 2_{u v}^{-n} \|^{f-f \|_{X}} d u d v \\
& \leqq \omega_{X}\left(f, 2^{-m}, 2^{-n}\right),
\end{aligned}
$$

which is the third inequality in (3.2).

The second inequality in (3.2) is trivial. have

We observe that for any polynomial $P \in P 2^{m}, 2^{n}$ and $(u, v) \in I_{m n}(0,0)$ we

$$
P(x \dot{+} u, \dot{y} \dot{v})=P(u, v) \text {. }
$$

Consequently, for such $u, v$

$$
\tau_{u v} f-f=\tau_{u v}(f-P)-(f-P) .
$$

Now, let $P$ be a best approximation to $f$ in $P_{2^{m}, 2^{n}}$. Then

$$
\omega_{X}\left(f ; 2^{-m}, 2^{-n}\right) \leqq 2\|f-P\|_{X}=2 E_{2^{m}, 2^{n}}(f ; X) .
$$

This is equivalent to the first inequality in (3.2).

The following corollary of Theorem 1 shows that the Lipschitz classes can be used to characterize functions by their rate of approximation by double Walsh polynomials.

COROLLARY 1. Let $j \in X$ and $\alpha, \beta>0$. Then the following five statements are equivalent:

(a) $f \in \operatorname{Lip}(\alpha, \beta ; X)$,

(b) $\left\|_{2^{m}, 2^{n}}(f)-\right\|_{X}=O\left(2^{-m \alpha}+2^{-n \beta}\right)$ as $m, n \rightarrow \infty$,

(c) $E_{2^{m}, 2^{n}}(f ; X)=O\left(2^{-m \alpha}+2^{-n \beta}\right)$ as $m, n \rightarrow \infty$,

(d) $E_{j k}(f ; X)=O\left(j^{-\alpha}+k^{-\beta}\right)$ as $j, k \rightarrow \infty$,

(e) $\omega_{X}\left(f ; 2^{-m}, 2^{-n}\right)=O\left(2^{-m \alpha}+2^{-n \beta}\right)$ as $m, n \rightarrow \infty$. .

PROOF. According to Theorem 1, (a) implies (b) and (c).

By definition,

$$
E_{j k}(f ; X) \leqq E_{i \tau}(f ; X) \text { whenever } j \geq i \text { and } k \geq \imath \text {. }
$$

Consequently, if

$$
2^{m} \leqq j<2^{m+1}, 2^{n} \leqq k<2^{n+1}, \text { and } m, n \geqq 0 \text {, }
$$

then

$$
E_{2^{m+1}}, 2^{n+1}(f ; X) \leqq E_{j k}(f ; X) \leqq E_{2^{m}, 2^{n}}(f ; X) .
$$

Hence it follows that (c) and (d) are equivalent.

By Theorem 1 and (3.7), (d) implies (e).

Finally, the fact that $\omega_{X}\left(f ; \delta_{1}, \delta_{2}\right)$ decreases as either $\delta_{1}$ or $\delta_{2}$ decreases shows that (e) and (a) are equivalent.

On closing, we note that Theorem 1 and corollary 1 are the multivariate extensions of the corresponding results by watari [9], proved for the cases $X=C_{W}(I)$ and $L^{p}(I), 1 \leqq p<\infty$. 
4. APPROXIMATION BY CESÀRO MEANS.

As is well-known, the first arithmetic means or cesàro means of series (3.1) are defined by

$$
\sigma_{m n}(f ; x, y):=\frac{1}{m n} \sum_{j=1}^{m} \sum_{k=1}^{n} S{ }_{j k}(f ; x, y), \quad m, n \cong 1 .
$$

It follows from (3.3) that

$$
\sigma_{m n}(f ; x, y)=\int_{0}^{1} \int_{0}^{1} f(x+u, y+v) K_{m}(u) K_{n}(v) d u d v
$$

where

$$
K_{m}(u):=\frac{1}{m} \sum_{j=1}^{m} D_{j}(u)
$$

is the Walsh-Fejer kernel. This kernel has the remarkable property of quasi-positiveness:

$$
\left\|K_{m}\right\|_{1}:=\int_{0}^{1}\left|K_{m}(u)\right| d u \leqq 2, m \geqq 1,
$$

first proved by Yano $[10]$. By $(2.1)$, we conclude that for any $f \in X$

$$
\left\|\sigma_{m n}(f)\right\|_{X} \leqq\left\|K_{m}\right\| 1\left\|K_{n}\right\|{ }_{1}\|f\|_{X} \leqq 4\|f\|_{X} .
$$

We estimate the rate of convergence when a function is approximated by the Cesàro means of its double Walsh-Fourier series.

THEOREM 2. For any $f \in X$ and $j, k \geqq 1$,

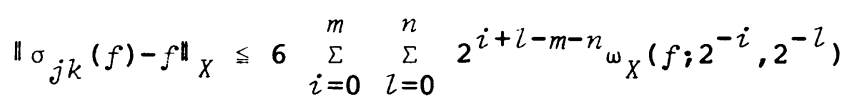

where $m$ and $n$ are defined in (3.6).

The next two corollaries are immediate consequences of Theorem 2 .

COROLLARY 2. (i) If $f \in L^{p}\left(I^{2}\right)$ for some $1 \leqq p<\infty$, then the cesàro means $\sigma_{j k}(f)$ of its double Walsh-Fourier series converge to $f$ in $L^{p}-$ norm.

(ii) If $f \in C_{W}\left(I^{2}\right)$, then the $\sigma_{j k}(f)$ converge to $f$ uniformly on $I^{2}$.

In statement (i), the case $p=1$ in really interesting. In

Section 6 , we will prove that, in the cases when $1<p<\infty$, even the rectangular partial sums $S_{j k}(f)$ converge to $f$ in $L^{p}$-norm (see Theorem 5 below). Statement (ii) is the multivariate extension of the corresponding result by Fine [4].

COROLLARY 3. If $f \in \operatorname{Lip}(\alpha, \beta ; X)$ for some $\alpha, \beta>0$, then

$$
\left\|\sigma_{j k}(f)-f\right\|_{X}= \begin{cases}O\left(j^{-\alpha}+k^{-\beta}\right) & \text { if } 0<\alpha, \beta<1, \\ O\left(j^{-1} \log j+k^{-\beta}\right) & \text { if } 0<\beta<1=\alpha, \\ O\left(j^{-1}+k^{-\beta}\right) & \text { if } 0<\beta<1<\alpha, \\ O\left(j^{-1} \log j+k^{-1} \log k\right) & \text { if } \alpha=\beta=1, \\ O\left(j^{-1}+k^{-1} \log k\right) & \text { if } 1=\beta<\alpha, \\ O\left(j^{-1}+k^{-1}\right) & \text { if } 1<\alpha, \beta .\end{cases}
$$

We note that corollary 3 is also the multivariate extension of the corresponding results by Yano [11] (proved for $0<\alpha<1$ and $1 \leqq p \leqq \infty$ ) and by Jastrebova [5] (proved for $\alpha=1$ and $p=\infty$ ). 
PROOF OF THEOREM 2. Keeping (3.6) in mind, we may write

$$
\begin{aligned}
& \sigma_{j k}(f)-f=\frac{2^{m+n}}{j k}\left(\frac{1}{2^{m+n}} \sum_{i=1}^{2^{m}} \sum_{i=1}^{2^{n}} S_{i l}(f)-f\right)
\end{aligned}
$$

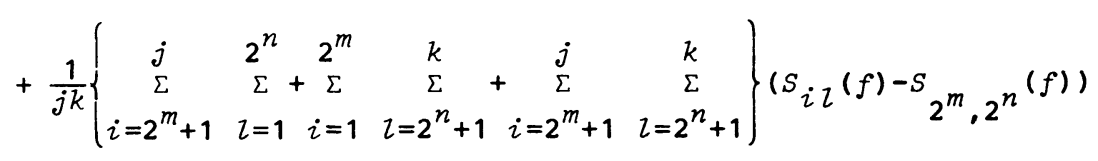

$$
\begin{aligned}
& +\left(1-\frac{2^{m+n}}{j k}\right)\left(S_{2^{m}, 2^{n}}(f)-f\right) \\
& =\frac{2^{m+n}}{j k}\left(\sigma_{2^{m}, 2^{n}}(f)-f\right)+\sigma_{m n}\left(f-S 2_{2^{m}, 2^{n}}(f)\right)+\left(1-\frac{2^{m+n}}{j k}\right)\left(S_{2^{m}, 2^{n}}(f)-f\right) . \\
& \text { Hence, by the triangle inequality and (4.1), } \\
& \|_{j k}(f)-\left.f\right|_{X} \leq\left|\sigma_{2^{m}, 2^{n}}(f)-f{ }_{X}+5\right| S_{2^{m}, 2^{n}}{ }^{(f)-f \|_{X}}
\end{aligned}
$$

Consequently, by Theorem 1 ,

$$
\left\|\sigma_{j k}(f)-f\right\|_{X} \leq\left\|_{2^{m}, 2^{n}}(f)-f\right\|_{X}+5 \omega_{X}\left(f ; 2^{-m}, 2^{-n}\right) .
$$

Now, we estimate the first quantity on the right-hand side of (4.5). To this end, we recall the representation

$$
K_{2^{m}}(u)=2^{-1}\left\{2^{-m} 2^{m}(u)+\sum_{i=0}^{m} 2^{i-m} 2^{m}\left(u+2^{-i-1}\right)\right\}, \quad u \in I
$$

(see, e.g., [8, p. 46, relation (iii)]). By (3.4), for $0 \leq i<m$

$$
D_{2^{m}}\left(u+2^{-i-1}\right)= \begin{cases}2^{m} & \text { if } u \in\left[2^{-i-1}, 2^{-i-1}+2^{-m}\right), \\ 0 & \text { otherwise; }\end{cases}
$$

and for $i=m$

$$
D_{2^{m}}\left(u \dot{+} 2^{-m-1}\right)=D_{2^{m}}(u)= \begin{cases}2^{m} & \text { if } u \in\left[0,2^{-m}\right), \\ 0 & \text { otherwise. }\end{cases}
$$

In particular, it follows that ${ }_{2^{m}}(u) \geq 0$ for all $u \in I$.

Similarly to (3.5), we apply again (2.1) and then by an elementary reasoning we obtain that

$$
\begin{aligned}
& I_{2^{m}, 2^{n}}(f)-\left.f\right|_{X} \leq \int_{0}^{1} \int_{0}^{1} \mid \tau_{u v}{ }^{f-f \mid}{ }_{X}{ }_{2^{m}}(u) K_{2^{n}}(v) d u d v
\end{aligned}
$$

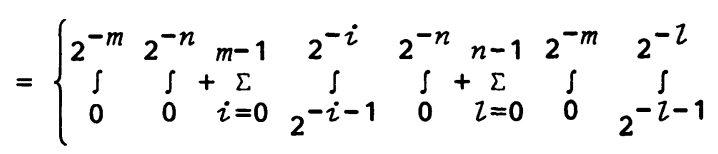

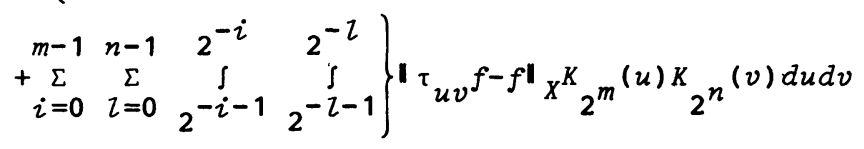

$$
\begin{aligned}
& \leqslant \omega_{X}\left(f ; 2^{-m}, 2^{-n}\right)+\sum_{i=0}^{m-1} 2^{i-m-1} \omega_{X}\left(f ; 2^{-i}, 2^{-n}\right)
\end{aligned}
$$




$$
\begin{aligned}
& +\sum_{l=0}^{n-1} 2^{i-n-1} \omega_{X}\left(f ; 2^{-m}, 2^{-l}\right) \\
& \sum_{i=0}^{m-1} \sum_{i=0}^{n-1} 2^{i-m-1} 2^{l-n-1} \omega_{X}\left(f ; 2^{-i}, 2^{-l}\right) \\
& \leqq \sum_{i=0}^{m} \sum_{l=0}^{n} 2^{i+l-m-n} \omega_{X}\left(f ; 2^{-i}, 2^{-l}\right) .
\end{aligned}
$$

Combining (4.5) and (4.6) yields (4.3).

5. APPROXIMATION BY DE LA VALLEE POUSSIN MEANS.

By Corollaries 1 and 3 , the rate of approximation by $\sigma_{j k}(f)$ is as good as by $S_{2^{m}, 2^{n}}(f)$ if $f \in \operatorname{Lip}(\alpha, \beta ; X)$ for some $0<\alpha, \beta<1$, where $m$ and $n$ are defined by $(3.6)$. However, the $\sigma_{j k}^{\prime} s$ are not projections from $x$ onto $P_{j k}$. These two important properties are satisfied by the de la Vallée Poussin means of series (3.1) defined by

$$
V_{m n}(f ; x, y):=\frac{1}{m n} \sum_{j=m+1}^{2 m} \sum_{k=n+1}^{2 n} S_{j k}(f ; x, y), \quad m, n \geq 1 .
$$

THEOREM 3. For any $f \in X$ and $m, n \geq 1$,

$$
\mid V_{m n}(f)-f \|_{X} \leqslant 37 E_{m n}(f ; X) \text {. }
$$

We note that in the univariate case, Bljumin [1], Esfahanizadeh and Siddiqi [3] studied de la vallée poussin means and obtained an inequality whose multivariate extension is (5.1).

PROOF. A routine computation shows that

$$
\begin{aligned}
v_{m n}(f ; x, y) & =s_{m n}(f ; x, y) \\
& +2 \sum_{j=m}^{2 m-1} \sum_{k=0}^{n-1}\left(1-\frac{j}{2 m}\right) a_{j k} w_{j}(x) w_{k}(y) \\
& +2 \sum_{j=0}^{m-1} \sum_{k=n}^{2 n-1}\left(1-\frac{k}{2 n}\right) a_{j k} w_{j}(x) w_{k}(y) \\
& +4 \sum_{j=m}^{2 m-1} \underset{k=n}{2 n-1}\left(1-\frac{j}{2 m}\right)\left(1-\frac{k}{2 n}\right) a_{j k} w_{j}(x) w_{k}(y) .
\end{aligned}
$$

Hence it follows immediately that for any $P \in P_{m n}$

$$
V_{m n}(P ; x, y)=P(x, y) \text {. }
$$

on the other hand, it is easy to check that

$$
V_{m n}(f)=4 \sigma_{2 m, 2 n}(f)-2 \sigma_{2 m, n}(f)-2 \sigma_{m, 2 n}(f)+\sigma_{m n}(f) .
$$

Consequently, by (4.2), for any $f \in X$ and $m, n \geq 1$ we have

$$
\left\|V_{m n}(f)\right\|_{X} \leq\left. 36\right|_{f} \|_{X} \text {. }
$$

Now, let $P$ be a best approximation to $f$ in $P_{m n}$. Then, combining (5.2) and (5.3) yields 


$$
\begin{aligned}
\left\|V_{m n}(f)-f\right\|_{X} & \leqq\left\|V_{m n}(f-P)\right\|_{X}+\|P-f\|_{X} \\
& \leqq 37\|P-f\|_{X}=37 E_{m n}(f ; X) .
\end{aligned}
$$

\section{ESTIMATION AND SATURATION PROBLEMS.}

(A) Theorem 1 says that the rate of approximation by the rectangular partial sums $S_{2^{m}, 2^{n}}(f)$ of the Walsh-Fourier series (3.1) is no worse than that by double Walsh polynomials from $P{ }_{2^{m}, 2^{n}}$ at all. As to approximation by $S_{m n}(f)$, we can ensure only a weaker rate in general.

THEOREM 4. For any $f \in X$ and $m, n \geqq 1$,

$$
\left\|S_{m n}(f)-f\right\|_{X} \leqq\left(1+\left\|D_{m}\right\|\left\|D_{n}\right\|_{1}\right) E_{m n}(f ; X) \text {. }
$$

This can be proved in a routine way. For the reader's convenience, we sketch it.

PROOF. Let $P$ be a best approximation to $f$ in $P_{m n}$. Since $S_{m n}(P)=P_{\text {, }}$ we may write that

$$
\left\|S_{m n}(f)-f\right\|_{X} \leqq\left\|S_{m n}(f-P)\right\|_{X}+\|P-f\|_{X} \cdot
$$

Taking into account $(3.3),(2.1)$, and the fact that $|\cdot|_{X}$ is translation invariant gives that

$$
\begin{aligned}
\left\|S_{m n}(f-P)\right\|_{X} & \leqq \int_{0}^{1} \int_{0}^{1}\left|\tau_{u v}(f-P) \|_{X}\right| D_{m}(u) D_{n}(v) \mid d u d v \\
& =\|f-P\|_{X} D_{m}\left\|D_{n}\right\|_{1} .
\end{aligned}
$$

Now, (6.1) follows from (6.2) and (6.3).

We note that

$$
\text { I } D_{m} \|_{1}=O(\log m)
$$

and this estimate is sharp (see, e.g. [8, p. 35]). In spite of this fact, estimate $(6.1)$ can be essentially improved in the particular case when $X=L^{p}\left(I^{2}\right), 1<p<\infty$. We will write $\mid \cdot I_{p}^{\prime}$ instead of $\|{ }_{L}^{p}\left(I^{2}\right) \cdot$

THEOREM 5. For any $1<p<\infty$, there exists a constant $\tilde{K}_{p}$ such that for any $f \in L^{p}\left(I^{2}\right)$ and $m, n \geq 1$ we have

$$
\left\|S_{m n}(f)-f\right\|_{p} \leqq \widetilde{K}_{p} E_{m n}\left(f ; L^{p}\left(I^{2}\right)\right) \text {. }
$$

Theorem 5 is ultimately a consequence of the following result by Paley [7]: For any $1<p<\infty$, there exists a constant $k_{p}$ such that for any $g \in L^{p}(I)$ and $m \geqq 1$ we have

$$
\left|S_{m}(g)\right|_{p} \leqq K_{p}|g|_{p}
$$

where this time

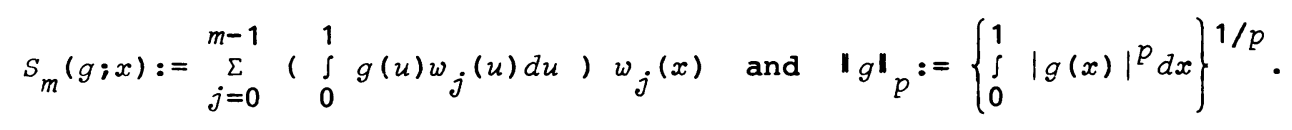

On the basis of (6.5) we will prove the following

LEMMA 2. For any $f \in L^{p}\left(I^{2}\right), 1<p<\infty$, and $m, n \geq 1$, 


$$
\left|S_{m n}(f)\right|_{p} \leqq K_{p}^{2}|f|_{p}
$$

PROOF. We may consider $f(x, y)$ as a function of $x$ for each fixed $y$ denoted by $g_{y}(x):=f(x, y)$. Observe that if $f \in L^{p}\left(I^{2}\right)$, then $g_{y} \in L^{p}(I)$ for almost all $y \in I$ and

$$
S_{m n}(f ; x, y)=S_{n}\left(S_{m}\left(g_{\underline{u}} ; x\right) ; y\right), \quad m, n \geq 1 .
$$

Furthermore, if $f \in L^{p}\left(I^{2}\right)$, then

$$
G_{m, x}(y):=S_{m}\left(g_{y} ; x\right)=\sum_{j=0}^{m-1}\left(\int_{0}^{1} g_{y}(u) w_{j}(u) d u\right) w_{j}(x) \in L^{p}(I)
$$

for all $m \geqq 1$ and for almost all $x \in I$.

Now, applying Fubini's theorem three times and the univariate inequality (6.5) twice provides (6.6) as follows

$$
\begin{aligned}
& \int_{0}^{1} \int_{0}^{1}\left|S_{m n}(f ; x, y)\right|^{p} d x d y \\
& =\int_{0}^{1}\left\{\left.\left.\int_{0}^{1}\right|_{k=0} ^{n-1}\left(\int_{0}^{1} G_{m, x}(v) w_{k}(v) d v\right) w_{k}(y)\right|^{p} d y\right\} d x \\
& \leqq \int_{0}^{1} K_{p}\left\{\int_{0}^{1}\left|G_{m, x}(y)\right|^{p} d y\right\} d x \\
& =K_{p} \int_{0}^{1}\left\{\int_{0}^{1}\left|\sum_{j=0}^{m-1}\left(\int_{0}^{1} g_{y}(u) w_{j}(u) d u\right) w_{j}(x)\right|^{p} d x\right\} d y \\
& \leqq K_{p} \int_{0}^{1} K_{p}\left\{\int_{0}^{1}\left|g_{y}(x)\right|^{p} d x\right\} d y \\
& =K_{p}^{2} \int_{0}^{1} \int_{0}^{1}|f(x, y)|^{p} d x d y .
\end{aligned}
$$

After these preliminaries, the proof of Theorem 5 is identical with that of Theorem 4, except that we use (6.6) instead of (6.3). In this way, we arrive at $(6.4)$ with $\tilde{K}_{p}:=1+K_{p}^{2}$.

Obviously, Theorem 5 implies the following

COROLLARY 4. If $f \in L^{p}\left(I^{2}\right)$ for some $1<p<\infty$, then the rectangular partial sums $S_{m n}(f)$ of its double Walsh-Fourier series converge to $f$ in $L^{P}$-norm.

Nevertheless, it seems to be very likely that estimate (6.1) is the best possible in general.

PROBLEM 1. Show that, in the cases when $X=L^{1}\left(I^{2}\right)$ or $C_{W}\left(I^{2}\right)$, there exists a function $f \in X$ such

$$
\underset{m, n \rightarrow \infty}{\lim \sup _{m}} \frac{\left|S_{m n}(f)-f\right| X}{\log m \log n}>0 .
$$

(B) We guess that Corollary 3 is also the best possible in the above sense. For example, we formulate this in connection with the fourth estimate in (4.4). 
PROBLEM 2. Show that, in the cases when $X=L^{1}\left(I^{2}\right)$ or $C_{W}\left(I^{2}\right)$, there exists a function $f \in \operatorname{Lip}(1,1 ; X)$ such that

$$
\lim _{m \rightarrow n \rightarrow \infty} \frac{\left.\right|_{\sigma_{m n}}(f)-f^{\prime} \mid}{m^{-1} \log m+n^{-1} \log n}>0 \text {. }
$$

In the univariate case, the corresponding result was proved by Jastrebova [5] with "lim" instead of "lim sup".

(C) Finally, we discuss the so-called saturation problem. We begin with the observation that the rate of approximation by the Cesàro means $\sigma_{m n}(f)$ to functions $f \in \operatorname{Lip}(\alpha, \beta)$ is not improved as $\alpha$ and $B$ increase beyond 1 . Indeed, the following is true.

THEOREM 6. If for some $f \in X$

$$
\left\|\sigma_{2^{n}, 2^{n}}(f)-f\right\|_{X}=o\left(2^{-n}\right) \text { as } n \rightarrow \infty \text {, }
$$

then $f$ is constant.

PROOF. Since

$$
{ }_{2^{n}, 2^{n}}(f ; X) \leqq \mathbf{I}_{2^{n}, 2^{n}}(f)-f^{\prime} X^{\prime}
$$

by hypothesis and Theorem 1, we have

$$
\text { I }_{2^{n}, 2^{n}}(f)-f \|_{X}=o\left(2^{-n}\right) \text { as } n \rightarrow \infty \text {. }
$$

Taking into account that

$$
2^{n}\left(S 2^{n}, 2^{n}(f ; x, y)-\sigma 2^{n}, 2^{n}(f ; x, y)\right)=\sum_{j=0}^{2^{n}-1} \sum_{k=0}^{n}\left(j+k-2^{-n} j k\right) a_{j k^{w}}(x) w_{k}(y) \text {, }
$$

by $(6.7)$ and $(6.8)$, we conclude that

$$
\lim _{n \rightarrow \infty}\left|\sum_{j=0}^{2^{n}-1} \sum_{k=0}^{n}\left(j+k-2^{-n} j k\right) a_{j k} w_{j}(x) w_{k}(y)\right|_{X}=0 \text {. }
$$

Since \|\|$_{1} \leqq\|\cdot\|_{X}$, it follows that

$$
\begin{aligned}
& \left|\left(j_{0}+k_{0}\right) a_{j_{0}, k_{0}}\right|
\end{aligned}
$$

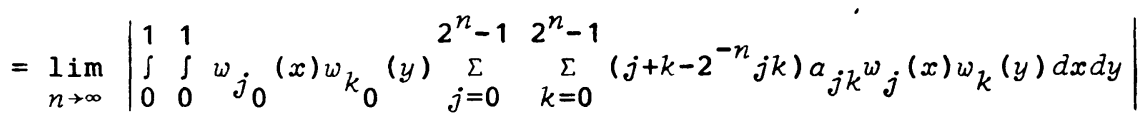

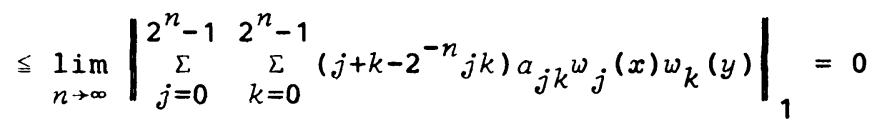

for all $j_{0}, k_{0} \geqq 0$ such that $\max \left(j_{0}, k_{0}\right) \geqq 1$. This implies that $a_{j_{0}, k_{0}}=0$ for all such pairs $j_{0}, k_{0}$, and therefore, $f=a_{00}$ is constant.

PROBLEM 3. How can one characterize those functions $f \in X$ such that

$$
\left\|\sigma_{j k}(f)-f\right\|_{X}=O\left(j^{-1}+k^{-1}\right) \text { ? }
$$

This is not known even in the univariate case. We conjecture that, in the special case when $j=k=2^{n}, X=C_{W}\left(I^{2}\right)$ or $L^{p}\left(I^{2}\right)$ for some $1 \leqq p<\infty$, we have 


$$
\operatorname{Io}_{2^{n}, 2^{n}}(f)-\left.f\right|_{X}=O\left(2^{-n}\right)
$$

if and only if

$$
\sum_{i=0}^{n} \sum_{l=0}^{n} 2^{i+l} \omega\left(f ; 2^{-i}, 2^{-i}\right)=o\left(2^{n}\right) .
$$

The "if" part follows from (4.6). The proof (or disproof) of the "only if" part is a problem.

ACKNOWLEDGEMENT: This work was partially supported by Grant No. 234 from the Hungarian National Foundation for Scientific Research.

\section{REFERENCES}

1. BLJUMIN, S.L. Certain properties of a class of multiplicative systems and problems of approximation of functions by polynomials with respect to those systems, Izv. Vuzov Mat. №. 4 (1967), 13-22 (Russian).

2. BUTZER, P.L. and NESSEL, R.J. Fourier analysis and approximations, Vol. 1, Birkhäuser, Basel and Academic Press, New York-London, 1971.

3. ESFAHANIZADEH, J. and SIDDIQI, A.H. On the approximation of functions by de la Vallée-Poussin mean of their Walsh-Fourier series, Aligarh Bull. Math. $\underline{8}$ (1978), 59-64.

4. FINE, N.J. On the Walsh functions, Trans. Amer. Math. Soc. 65 (1949), 372-414.

5. JASTREBOVA, M.A. On approximation of functions satisfying the Lipschitz condition by arithmetic means of their Walsh-Fourier series, Mat. Sbornik 71 (113) (1966), 214-226 (Russian).

6. KATZNELSON, Y. Harmonic analysis, John Wiley and Sons, New York, 1968.

7. PALEY, R.E.A.C. A remarkable system of orthogonal functions, Proc. London Math. Soc. 34 (1932), 241-279.

8. SCHIPP, F., WADE, W.R. and SIMON, P. Walsh series. An introduction to dyadic harmonic analysis, Akadémiai Kiadó, Budapest, 1990.

9. WATARI, C. Best approximation by Walsh polynomials, Tôhoku Math. J. 15 (1963), 1-5.

10. YANO, SH. On Walsh series, Tôhoku Math. J. $\underline{3}$ (1951), 223-242.

11. YANO, SH. On approximation by Walsh functions,

Proc. Amer. Math. Soc. 2 (1951), 962-967. 


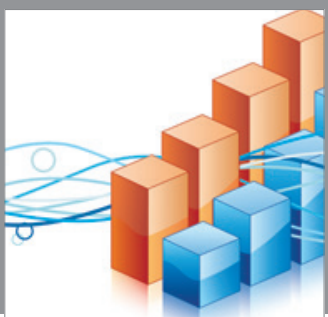

Advances in

Operations Research

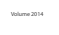

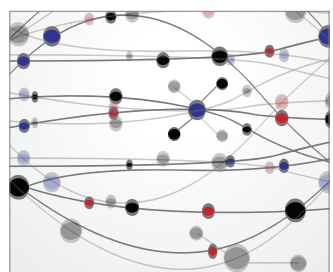

\section{The Scientific} World Journal
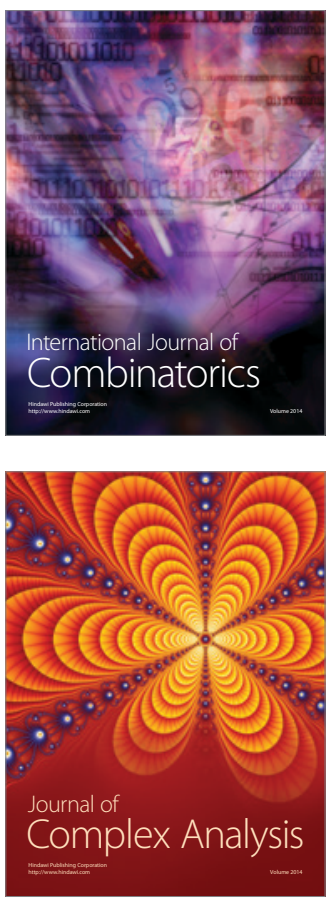

International Journal of

Mathematics and

Mathematical

Sciences
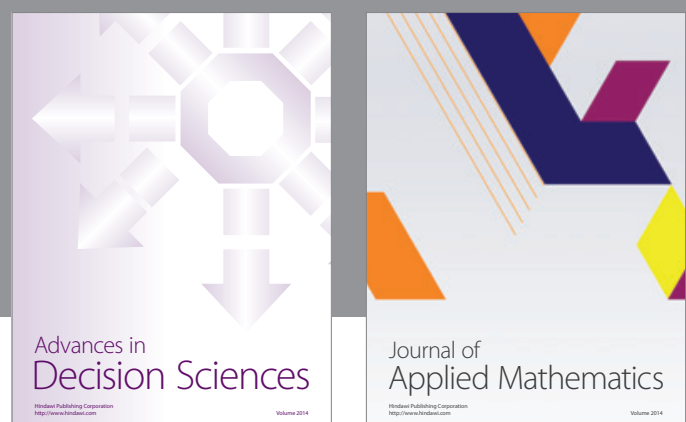

Journal of

Applied Mathematics
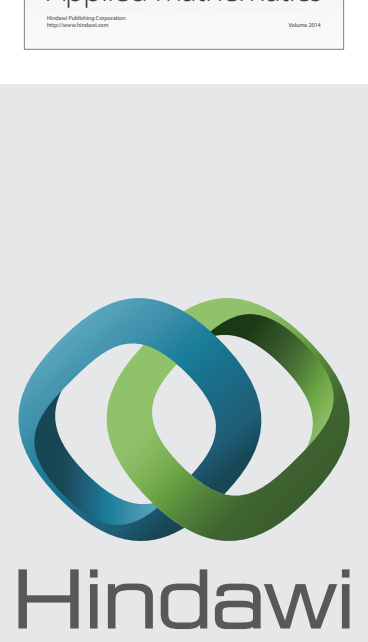

Submit your manuscripts at http://www.hindawi.com
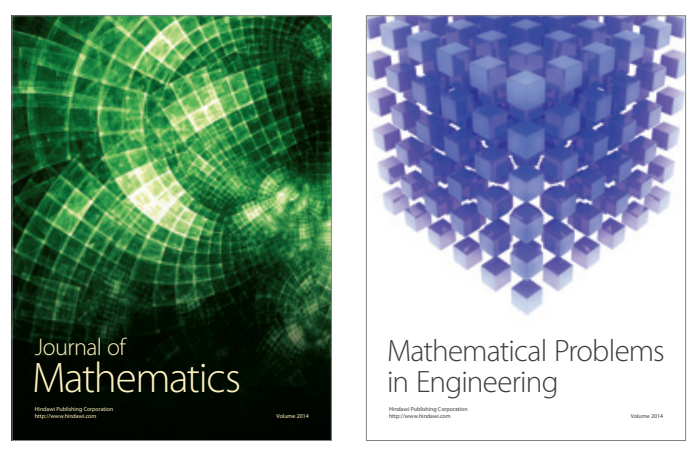

Mathematical Problems in Engineering
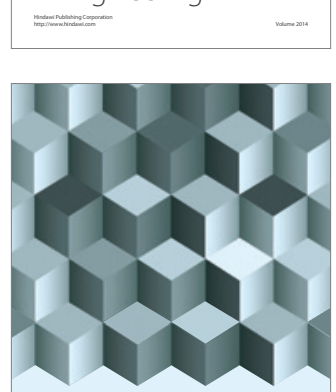

Journal of

Function Spaces
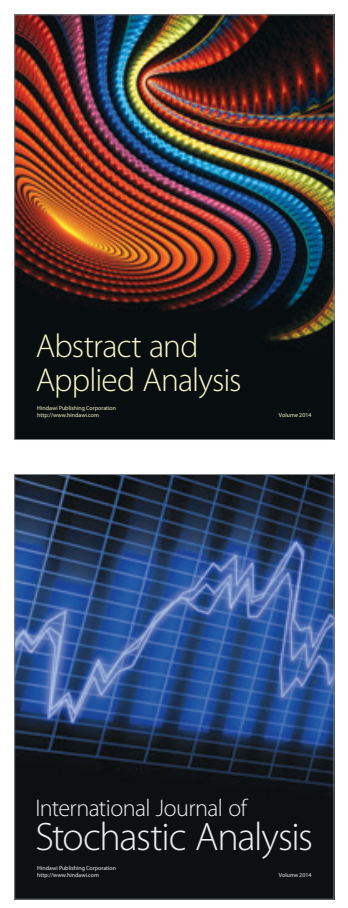

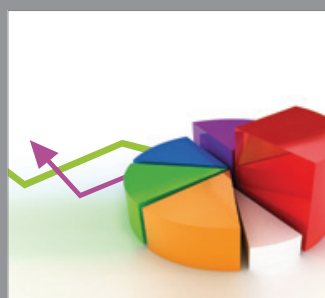

ournal of

Probability and Statistics

Promensencen
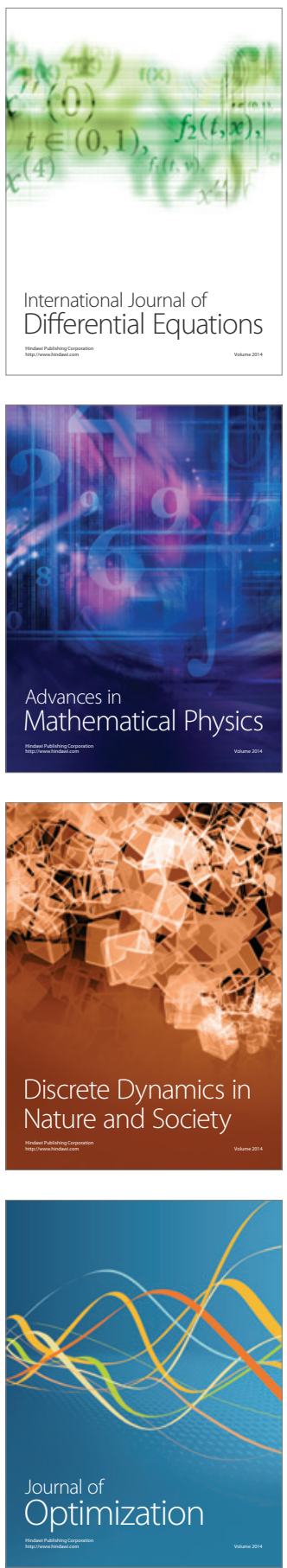